\title{
Warum lebensweltlich deutschsprachige Schülerinnen und Schüler von einem sprachenintegrativen Deutschunterricht profitieren - empirische Erkenntnisse
}

\author{
Anja Wildemann • Lena Bien-Miller (D)
}

Eingegangen: 26. Februar 2021 / Angenommen: 23. November 2021 / Online publiziert: 7. Januar 2022 (C) Der/die Autor(en) 2022

Zusammenfassung In einem sprachenintegrativen Deutschunterricht werden die Kontaktsprachen der Schülerinnen und Schüler systematisch und zielorientiert einbezogen und für das sprachliche Lernen genutzt. Zur Wirksamkeit eines solchen sprachenintegrativen (Deutsch-)Unterrichts auf die Reflexions- und Analysefähigkeiten lebensweltlich einsprachiger und mehrsprachiger Schülerinnen und Schüler liegen bislang keine Studien vor. Ein Zusammenhang zwischen der sprachenintegrativen und -vergleichenden Arbeit und der Entwicklung von Sprachbewusstheit einund mehrsprachiger Schülerinnen und Schüler wird zwar immer wieder vermutet, ist empirisch jedoch nicht belegt. Daran anknüpfend fokussiert der vorliegende Beitrag die Frage, ob sich ein sprachenintegrativer Deutschunterricht, in dem systematisch andere Sprachen vergleichend zur Zielsprache Deutsch thematisiert werden, positiv auf sprachbezogene Reflexions- und Analysefähigkeiten d.h. auf die Sprachbewusstheit, lebensweltlich ein- und mehrsprachiger Schülerinnen und Schüler auswirkt.

Schlüsselwörter Sprachenintegrativer Deutschunterricht · Lebensweltlich ein- und mehrsprachige Schülerinnen und Schüler · Sprachbewusstheit · Sprachreflexion · Sprachanalyse

\footnotetext{
Prof. Dr. Anja Wildemann ( $\triangle)$

Arbeitsbereich Grundschulpädagogik, Institut für Bildung im Kindes- und Jugendalter, Universität Koblenz-Landau, August-Croissant-Str. 5, 76829 Landau in der Pfalz, Deutschland

E-Mail: wildemann@uni-landau.de

Dr. Lena Bien-Miller

FB05 Sprache, Literatur, Kultur, Institut für Germanistik, Justus-Liebig-Universität Gießen, Otto-Behaghel-Straße 10B, 35394 Gießen, Deutschland

E-Mail: Lena.Bien-Miller@germanistik.uni-giessen.de
} 


\title{
Why German-speaking students benefit from language-integrative German classes-empirical findings
}

\begin{abstract}
In a language-integrative German classroom, students' contact languages are systematically and purposefully included and thus used for language learning. However, there are hardly any studies on the effectiveness of language-integrative (German) teaching on reflective faculties and analytical skills as linguistic sub-competencies of language awareness of lifelong monolingual and multilingual students. Although positive effects are repeatedly assumed, they have not been empirically proven. The question of whether language-integrative German instruction in which other languages are systematically examined in comparison to the target language German has a positive effect on the competencies of monolingual and multilingual students in the area of language reflection and analysis, will be addressed in this article.
\end{abstract}

Keywords Language integrating classes · Plurilingual students · German language classes $\cdot$ Linguistic awareness $\cdot$ Language reflection $\cdot$ Language analysis

\section{Einleitung}

Aktuell besteht eine Schieflage zwischen verschiedenen Konzepten für die Integration von Sprachen in den Deutschunterricht und deren empirischer Fundierung. So liegt zwar eine Reihe von konzeptionellen Überlegungen vor (u. a. Kurtz et al. 2014; Oomen-Welke 2017; Reich und Krumm 2013), diese sind jedoch zumeist bis dato weder evaluiert noch auf ihre Wirksamkeit hin überprüft worden. Vor diesem Hintergrund wird im Forschungsprojekt MehrSprachen ein sprachenintegratives Konzept entwickelt und im Hinblick auf seine Wirksamkeit für die Entwicklung von Sprachbewusstheit lebensweltlich ${ }^{1}$ ein- und mehrsprachiger Schülerinnen und Schüler untersucht. Unter einem sprachenintegrativen Deutschunterricht wird dabei ein Unterricht verstanden, ,in dem die unterschiedlichen sprachlichen Voraussetzungen und Vorkenntnisse der Schülerinnen und Schüler, wie sie beispielsweise durch die Herkunftssprache oder die Berührung mit Fremdsprachen bedingt sind, als Ressource betrachtet und genutzt werden. Ziel des Unterrichts ist es, diese sprachlichen Ressourcen lernförderlich in den Unterricht einzubeziehen“ (Wildemann, Andronie, Bien-Miller und Krzyzek 2020, S. 183).

Im Forschungsprojekt „MehrSprachen“ wurde das Konzept eines sprachenintegrativen Deutschunterrichts im Rahmen einer sechsmonatigen Intervention im Deutschunterricht der Grundschule (Klasse 3) erprobt, und im Hinblick auf seine Wirksamkeit für die Entwicklung von Sprachbewusstheit untersucht. Der Vergleich lebensweltlich mehrsprachiger und einsprachiger Lernerinnen und Lerner hat Unerwartetes zutage gebracht: In Bezug auf die Entwicklung von Sprachbewusstheit

\footnotetext{
1 Busch (2016) definiert lebensweltliche Mehrsprachigkeit in Abgrenzung zu „,beigebrachten Sprachen“ (Busch 2016, S. 42). Lebensweltliche Mehrsprachigkeit umfasst dabei diejenigen Sprachen, die im Alltag eines Menschen eine funktionale Bedeutung haben (siehe auch Grosjean 2020).
} 
profitieren insbesondere lebensweltlich einsprachige Lernerinnen und Lerner von einem sprachenintegrativen Deutschunterricht. Diese Erkenntnis stellt die bisher nicht selten vertretene Annahme, dass vor allem lebensweltlich mehrsprachige Lernerinnen und Lerner von dem Einbeziehen ihrer Sprachen in den Unterricht profitieren (Oomen-Welke 2017; Rösch 2017), dies aber für lebensweltlich einsprachige Lernerinnen und Lerner nicht gelte (Esser 2006²), auf den Kopf und bietet ein Potenzial für einen Deutschunterricht, in dem sprachliche Diversität Ausgangs- und Zieldimension ist.

\section{Theoretischer und empirischer Hintergrund}

\subsection{Sprachbewusstheit als Fähigkeit zur Sprachreflexion und -analyse}

Unter Sprachbewusstheit wird im Allgemeinen die Fähigkeit verstanden, Sprache zum Gegenstand des Denkens und Sprechens zu machen und dabei auf das Wissen über Sprache, das metasprachliche Wissen, zurückzugreifen, es zu nutzen und gleichzeitig weiterzuentwickeln. Im Fachdiskurs wird das Konstrukt Sprachbewusstheit aus unterschiedlichen Perspektiven und unter Verwendung unterschiedlicher Termini umrissen: Sprachbewusstheit/Sprachbewusstsein (Andresen 1985; Eichler 2007; Wildemann 2013), Sprachreflexion (Budde 2012; Kuyumcu 2014), Sprachaufmerksamkeit (Oomen-Welke 2010) sowie Language Consciousness/Awareness (Hawkins 1984; James und Garrett 1992) und (Meta)linguistic Ability bzw. Awareness (Bialystok 1991; Bialystok und Ryan 1985; Karmiloff-Smith 1992). Die divergente Begriffsverwendung geht zum großen Teil auf unterschiedliche Forschungskontexte zurück, in denen Sprachbewusstheit modelliert und operationalisiert wurde: bspw. im Rahmen der sprachlichen (Karmiloff-Smith 1992; Gombert 1992), der kognitiven (Hakes 1980; Schöler 1987) oder der sprachlich-kognitiven Entwicklung (Bialystok und Ryan 1985; Waller 1988), im Rahmen des Schriftspracherwerbs (Andresen 1985) oder der Didaktik des schulischen Deutschunterrichts (Eichler 2007). Mittlerweile besteht im nationalen und internationalen Diskurs ein Konsens darüber, dass Sprachbewusstheit sowohl im Zuge der kognitiven als auch der sprachlichen Entwicklung entsteht (Andresen 1985; Gornik 1989, 2014; Karmiloff-Smith 1992; Gombert 1992) und sich durch den schulischen Sprachunterricht maßgeblich weiter entwickelt (Karmiloff-Smith 1992; Ulrich 2001; Andresen 1985). In der Regel wird dem Zwei-Komponenten-Modell von Bialystok und Ryan (1985) folgend eine zweifaktorielle Struktur des Konstrukts Sprachbewusstheit angenommen (Waller 1988; Krafft 2014; Bialystok 1986, 1988, 1999; Cromdal 1999). Dabei wird davon ausgegangen, dass für die Herausbildung von Sprachbewusstheit zwei sprachlichkognitive Prozesse verantwortlich sind: analysis of linguistic knowledge und control of linguistic processing (Bialystok 1991, S. 116). Während die control-Komponente sich auf die kontrollierte Aufmerksamkeitssteuerung bei der Sprachverarbeitung

\footnotetext{
${ }^{2}$ Esser (2006) bezieht sich auf den Zusammenhang von Bilingualität und Schulerfolg und schreibt dabei den Erstsprachen der Kinder in Anlehnung an Studien aus dem anglo-amerikanischen Raum eine „bedingte Wirkung" (S. 384) zu.
} 
bezieht und sich bspw. in Selbst- und Fremdkorrekturen zeigt, bezeichnet die analysis-Komponente die den Spracherwerb begleitende Transformation des impliziten sprachlichen Wissens in explizite, metasprachliche Wissensbestände.

Das Landauer Konstrukt von Sprachbewusstheit (Wildemann et al. 2018) orientiert sich ebenfalls an dem Zwei-Komponenten-Modell von Bialystok und Ryan (1985) und am Entwicklungsmodell von Karmiloff-Smith (1986, 1992). Als Indikator für Sprachbewusstheit werden metasprachliche Äußerungen angesehen, in denen Sprache zum Gegenstand des Denkens und Sprechens gemacht wird und die dahinterliegenden sprachbezogenen Wissensbestände offenbaren (Bialystok 2001; Karmiloff-Smith 1992; Waller 1988). Mit Bezug auf Karmiloff-Smith $(1986,1992)$ wird davon ausgegangen, dass Sprachbewusstheit aus sprachlichen Erfahrungen erwächst, mit kognitiven Kompetenzen einhergeht und sich durch angeleitete metasprachliche Aktivitäten im Unterricht positiv beeinflussen lässt. Sprachbewusstheit wird darüber hinaus eine Brückenfunktion für die Herausbildung der Teilfertigkeiten Hören, Lesen, Sprechen und Schreiben zugesprochen. Vor allem aus dem anglo-amerikanischen und kanadischen Kontext werden signifikante Zusammenhänge zwischen Sprachbewusstheit und Lesen, Schreiben und Sprechen berichtet (bspw. MarinovaTodd et al. 2013; Mimeau et al. 2019; Mokhtari und Thompson 2006).

\subsection{Sprachbewusstheit und explizite Sprachthematisierung}

In Forschung und Didaktik wird angenommen, dass Sprachbewusstheit durch unterrichtliche Sprachthematisierung, d.h. durch das Lösen von Sprache aus ihren unmittelbaren Verwendungskontexten und das Richten der Aufmerksamkeit auf formale und funktionale Aspekte von Sprache (vgl. Gornik 2010, S. 233) weiterentwickelt und gefördert werden kann.

Diese Annahmen wurden jedoch bislang nicht empirisch belegt (vgl. auch BienMiller und Wildemann 2020; Wildemann et al. 2020a). Im anglo-amerikanischen Diskurs wird die Frage nach der Wirksamkeit expliziter Sprachthematisierung im Kontext der Diskussion über die Durchlässigkeit der expliziten und impliziten Wissensformen beim gesteuerten Fremdspracherwerb diskutiert (Ellis 2008). Hier wird unter Bezugnahme auf die weak-interface-Hypothese (Ellis 2008) davon ausgegangen, dass das explizite Thematisieren von sprachlichen Funktionen und Formen positive Auswirkungen auf die Entwicklung von impliziten und expliziten sprachlichen und metasprachlichen Wissensbeständen hat. Im deutschsprachigen Raum weisen einige Studien aus dem DaZ-Kontext in ebendiese Richtung. So zeigt Rösch 2014 in ihrer Interventionsstudie, dass explizite Thematisierung von Sprache zur Verbesserung von Grammatikkönnen und der Leseflüssigkeit führt (Rösch 2014). Für den Englischunterricht konnte die DESI-Studie durch Videoanalysen einen Zusammenhang zwischen sprachbewusstem Unterricht und Lernzuwachs der Schülerinnen und Schüler belegen (Klieme und Rakoczy 2008). Inwieweit diese Resultate auf die Wirksamkeit von Sprachvergleich und -reflexion in einem sprachenintegrativen Deutschunterricht der Grundschule übertragen werden können, ist jedoch bislang noch ungeklärt. 


\subsection{Sprachbewusstheit und Mehrsprachigkeit}

Mehrsprachigkeit, d.h. die Fähigkeit, mehr als eine Sprache in alltäglichen kommunikativen Kontexten zu nutzen, wird im nationalen und internationalen Forschungsdiskurs als eine Einflussgröße auf Sprachbewusstheit angesehen. Allen voran sind hier die Arbeiten von Bialystok $(1987,1991)$ zu nennen, die gezeigt hat, dass mehrsprachige Kinder ihren monolingualen Altersgenossen sowohl bei control- als auch bei analysis-Aufgaben überlegen sind (Bialystok 1987, 2017). Diese Zusammenhänge werden von Galambos und Hakuta (1988) sowie von Cromdal (1999) bestätigt. Im deutschsprachigen Raum sind die Ergebnisse der bis dato vorliegenden Studien weniger eindeutig. Während Krafft (2014) feststellt, dass lebensweltlich einsprachige Kinder zum Ende der Grundschulzeit in beiden Kategorien (analysis und control) signifikant besser als mehrsprachige abschneiden, berichten Hopp et al. (2017) von Vorteilen der mehrsprachigen Kinder im Bereich control. Das Projekt „Sprachkompetenzen und Sprachbewusstheit" konnte zeigen, dass lebensweltlich mehrsprachige Schülerinnen und Schüler in der zweiten Hälfte der Grundschulzeit im Bereich analysis signifikant höhere Sprachbewusstheitswerte als lebensweltlich einsprachige zeigen. Sie stehen zudem in einem signifikanten Zusammenhang mit dem Niveau der Mehrsprachigkeit (d.h. mit den Sprachkompetenzen in den beiden Sprachen) stehen (Bien-Miller, Akbulut und Wildemann 2017). Einige wenige Untersuchungen zu Language Awareness (z. B. Bialystok 1987; Fehling 2005; James und Garrett 1992; Jessner 2007; Svalberg 2012), Translanguaging (z. B. García 2009; Hornberger und Holly 2012) sowie zu Lernerstrategien (z. B. Hattie et al. 1996; Tragant und Victori 2012) lassen ebenfalls vorsichtige Schlüsse zu, dass Mehrsprachigkeit als eine Ressource zum Aufbau von Sprachbewusstheit genutzt werden kann. In eine ähnliche Richtung weisen die Ergebnisse von Untersuchungen, die sich mit Teilaspekten von Sprachbewusstheit und der Rolle von Mehrsprachigkeit in diesem Rahmen beschäftigen (bspw. Galambos und Goldin-Meadow (1990), Galambos und Hakuta (1988) sowie Ricciardelli (1992) berichten positive Effekte von Mehrsprachigkeit auf die syntaktische Bewusstheit).

Inwiefern die Forschungsergebnisse, die von Mehrsprachigkeit ausgehende Vorteile für Sprachbewusstheit belegen, auf die Unterrichtspraxis übertragbar sind, bleibt aufgrund der unzureichenden Forschungslage unklar. Ebenfalls unklar ist, ob lebensweltlich einsprachige und mehrsprachige Schülerinnen und Schüler gleichermaßen von einem sprachenintegrativen Deutschunterricht profitieren. An dieser Fragestellung setzt das Projekt MehrSprachen an, indem es Effekte eines sprachenintegrativen Unterrichts auf die Entwicklung von Sprachbewusstheit im Grundschulalter untersucht.

\section{Folgerungen und Hypothesen}

Aus den theoretischen Überlegungen ergeben sich folgende Prämissen für die vorliegende Studie. Sprachbewusstheit wird als Fähigkeit verstanden, formale und funktionale Aspekte von Sprache zum Gegenstand des Denkens zu machen. Da diese nicht unmittelbar erfasst werden kann, werden metasprachliche Äußerungen, in 
denen Sprache vergegenständlicht wird und die dahinterliegende sprachbezogene Wissensbestände offenbaren, als Indikator von Sprachbewusstheit angesehen (vgl. Andresen 1985; Gornik 1989, 2010; Waller 1988). Bezugnehmend auf die Forschungsergebnisse, laut derer Mehrsprachigkeit eine Ressource für die Entwicklung von Sprachbewusstheit eines mehrsprachigen Individuums darstellt und unter Berücksichtigung der Befunde, die auf tendenziell positive Auswirkungen expliziter Sprachthematisierung auf die Sprachbewusstheit hindeuten, wird ein positiver Zusammenhang zwischen der Integration und der Nutzung von Mehrsprachigkeit im Rahmen unterrichtlicher Sprachthematisierung und der Entwicklung von Sprachbewusstheit angenommen. Vor diesem Hintergrund wird folgende Forschungsfrage verfolgt:

Wie wirkt sich ein sprachenintegrativer und -reflexiver Deutschunterricht auf Sprachbewusstheit von lebensweltlich ein- und mehrsprachigen Schülerinnen und Schülern am Ende der Grundschulzeit aus?

Auf Basis der dargestellten Forschungslage wird der Annahme gefolgt, dass sich Sprachbewusstheit von Schülerinnen und Schülern in der zweiten Hälfte der Grundschule durch einen sprachenintegrativen Deutschunterricht steigern lässt. Mit Blick auf lebensweltlich mehrsprachige Schülerinnen und Schüler wird auf der Grundlage bisheriger Studien zudem angenommen, dass Mehrsprachige stärker von einem sprachenintegrativen Deutschunterricht profitieren als ihre lebensweltlich einsprachigen Altersgenossinnen und -genossen. Demzufolge werden folgende Hypothesenformuliert und überprüft:

Hypothese 1 In der Experimentalgruppe (mit Intervention) erreichen Schülerinnen und Schüler höhere Sprachbewusstheitswerte als in der Kontrollgruppe (ohne Intervention).

Hypothese 2 Lebensweltlich mehrsprachige Schülerinnen und Schüler profitieren von einer Intervention in Bezug auf Sprachbewusstheit mehr als lebensweltlich einsprachige Schülerinnen und Schüler.

\section{Forschungsdesign}

\subsection{Anlage der Studie und Instrumente}

Die Untersuchung folgt einem Experimental-Kontrollgruppendesign mit drei Messzeitpunkten. Zu Studienbeginn wurde eine Hybrid-Delphi-Studie (siehe Abb. 1) mit Expertinnen und Experten aus Wissenschaft und Unterrichtspraxis durchgeführt (vgl. Andronie et al. 2019). Unter Berücksichtigung der sich durch ihre Auswertung herauskristallisierten Bedarfe aus der Unterrichtspraxis und in Anlehnung an Oomen-Welkes (2017) Didaktik der Vielsprachigkeit ergaben sich für das Konzept eines sprachenintegrativen Deutschunterrichts zwei didaktische Säulen: 1. Explizite Sprachthematisierung, bei der Sprachen systematisch und integrativ fokussiert werden (vgl. Gornik 2010). 2. Funktionale Sprachreflexion, die Sprachen unter Nut- 


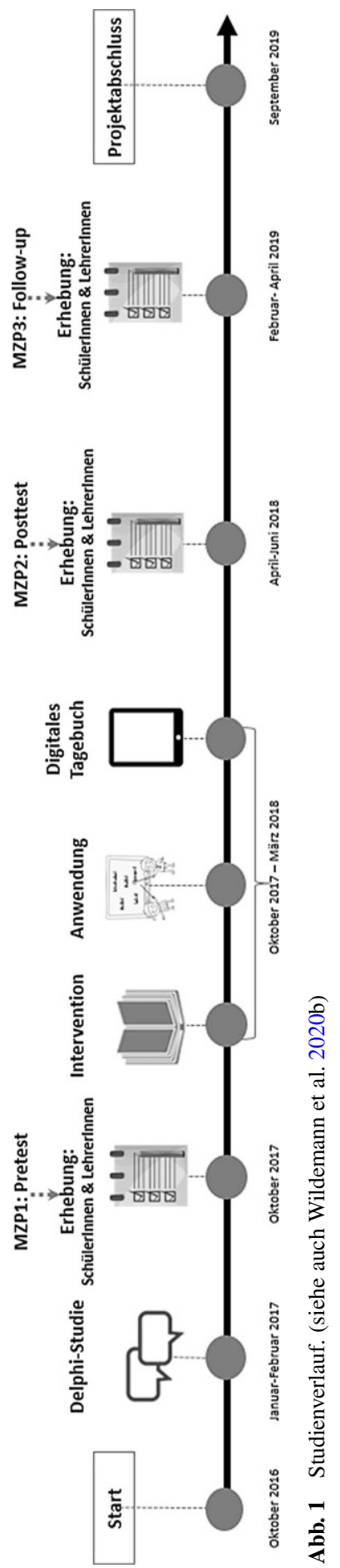


zung lebensweltlicher und durch den Fremdsprachenunterricht erzeugter emergenter Mehrsprachigkeit thematisiert und analysiert. Das Konzept bildete die Basis für eine sechsmonatige Fortbildung für die Lehrkräfte der Experimentalgruppe $(N=18)$, die sich aus $24 \mathrm{~h}$ Fortbildungszeit und Erprobungsphasen im eigenen Unterricht zusammensetzte. In einem digitalen Tagebuch wurde die Anwendung des sprachenintegrativen Deutschunterrichts durch die Lehrkräfte dokumentiert (Wildemann, Andronie, Bien-Miller und Krzyzek 2020).

Das Design der Studie im Zeitverlauf stellt Abb. 1 dar.

Die Erhebung von Schülerdaten erfolgte an drei Messzeitpunkten. Zum ersten Messzeitpunkt, im Vorfeld der Intervention, wurden die Hintergrundvariablen Sprachkompetenzen (Verfahren Tulpenbeet (Reich et al. 2008)), allgemeine kognitive Fähigkeiten (CFT 20-R (Weiß 2005)) sowie demografische Daten der Schülerinnen und Schüler erhoben. Zum zweiten und zum dritten Messzeitpunkt wurde direkt nach der Intervention und sechs Monate danach Sprachbewusstheit mit dem Elizitationsverfahren M-SPRA (Wildemann et al. 2016) erfasst.

\subsection{Operationalisierung und Elizitation von Sprachbewusstheit}

Sprachbewusstheit, als Fähigkeit, metasprachliche Handlungen zu vollziehen, wird in der vorliegenden Studie als die Anzahl eingebrachter metasprachlicher Äußerungen im Zuge des Arbeitens mit dem Verfahren M-SPRA (Wildemann et al. 2016) operationalisiert. M-SPRA elizitiert in Anlehnung an die Methode des konkurrenten Probings (vgl. Woolley et al. 2004) verbale Daten durch Aufforderung der Schülerinnen und Schüler, ihre Gedankengänge bei der Lösung einer sprachvergleichenden Aufgabe (Reflexionsimpuls) zu verbalisieren. Bei der Entwicklung der Reflexionsimpulse wurde zum einen eine größtmögliche Standardisierung angestrebt, um die Vergleichbarkeit der Schülerleistungen zu gewährleisten, zum anderen eine größtmögliche Offenheit der Aufgabenstellung, um ein möglichst breites linguistisches Spektrum in den metasprachlichen Äußerungen zu erhalten. Als eine sprachliche Vorlage nutzt das Verfahren eine mehrsprachige Software (,My First Stories“ 2013, Oldenbourg Verlag), die das Lesen und Hören von kindgerechten Geschichten (hier: „Maddox, der Magier“ und „Die Geschichte vom Eis“) in fünf Sprachen (Deutsch, Englisch, Spanisch, Russisch, Türkisch) ermöglicht und die Option bietet, jederzeit zwischen den Sprachen zu wechseln. Insgesamt werden zwei Reflexionsimpulssets (sprachvergleichende Aufgabenstellungen für die 3. bzw. 4. Klassenstufe) eingesetzt, die jeweils fünf Impulsaufgaben beinhalten, welche wiederum jeweils auf unterschiedliche linguistische Ebenen abzielen. Die Impulssets für Klassenstufen drei und vier unterscheiden sich voneinander in der Explizitheit der anvisierten metasprachlichen Wissensbestände: Das Set für die dritte Klassenstufe elizitiert in erster Linie Wissensbestände, die durch die selbständige gedankliche Auseinandersetzung mit Sprache entstehen. Für die vierte Klassenstufe werden darüber hinaus vor allem im Unterricht erworbene metasprachliche Kenntnisse erfasst (Tab. 1).

Der Ablauf des Verfahrens lässt sich folgenderweise beschreiben: Zwei Kinder bilden ein Interaktionsteam (Tandem) und bedienen die mehrsprachige Software. Während der Rezeption der Texte setzt eine geschulte Testleitung an den dafür vorgesehenen Stellen den entsprechenden Reflexionsimpuls. Die Kinder interagieren mit 
Tab. 1 Beispiel-Impulse für die 3. und 4. Klassenstufe

\begin{tabular}{lll}
\hline $\begin{array}{l}\text { Ling. } \\
\text { Ebene }\end{array}$ & MZP 2 & MZP 3 \\
\hline $\begin{array}{l}\text { Pragma- } \\
\text { tisch }\end{array}$ & $\begin{array}{l}\text { Was machen die Kinder da? (Streiten } \\
\text { die miteinander?) } \\
\text { Woran merkt man das? Was will denn }\end{array}$ & $\begin{array}{l}\text { Schaut euch mal diesen Text an. Was meint ihr, } \\
\text { was für ein Text das ist? } \\
\text { Woran habt ihr erkannt, dass es ein Rezept ist? }\end{array}$ \\
$\begin{array}{l}\text { Marnie von Maddox? Und was will er? } \\
\text { Morpho- }\end{array}$ & $\begin{array}{l}\text { Zählt doch mal nach. Wie viele Groß- } \\
\text { logisch }\end{array}$ & $\begin{array}{l}\text { Schaut euch diese Wörter hier an (auf Nomen } \\
\text { buchstaben gibt es auf dieser Seite? }\end{array}$ \\
& $\begin{array}{l}\text { Jetzt sucht euch eine zweite Sprache } \\
\text { aus, wie ist es da? Wo sind die ganzen } \\
\text { diese Wörter nennt? Woran kann man Nomen } \\
\text { erkennen? (Ins Türkische wechseln lassen.) Könnt } \\
\text { Großbuchstaben hin? Könnt ihr das } \\
\text { erklären? }\end{array}$ & $\begin{array}{l}\text { ihr auch hier Nomen finden? Woran habt ihr hier } \\
\text { die Nomen erkannt? }\end{array}$ \\
\hline
\end{tabular}

der Testleitung und untereinander und verbalisieren dadurch ihre sprachbezogenen Annahmen, Vorstellungen, Hypothesen etc. Diese metasprachlichen Interaktionen werden videographiert, vollständig transkribiert und inhaltsanalytisch ausgewertet. Die Gesamtzahl eingebrachter metasprachlicher Äußerungen bildet den Globalwert für Sprachbewusstheit, der für die statistischen Analysen genutzt wird.

\subsection{Stichprobe}

Insgesamt haben 409 Schülerinnen und Schüler aus 21 Grundschulen der dritten und vierten Klassenstufe an der Studie teilgenommen. Grunddaten zu Alter, Geschlecht, Geburtsort des Kindes und der Eltern, der Sprachverwendung innerhalb der Familie bzw. Peergroup etc. wurden mithilfe eines Schülerfragebogens ermittelt. Da keine komplexeren Konstrukte wie der soziokulturelle oder sozioökonomische Hintergrund erhoben wurden, erwies sich die direkte Befragung der Kinder als effizient. Die Vergleichbarkeit der Daten kann trotz Fehlens sozioökonomischer Daten aufgrund der Größe der Stichprobe und der ausgewogenen Verteilung auf lebensweltlich einsprachige und lebensweltlich mehrsprachige Kinder auf Schulebene als weitgehend erfüllt betrachtet werden. Auf einen hohen Grad an Randomisierung der Stichprobe weist zudem die Normalverteilung hin, die im Bereich der Sprachkompetenzen im Deutschen und der kognitiven Grundfähigkeiten erreicht wurde. Tab. 2 zeigt die Zusammensetzung der Stichprobe hinsichtlich des Sprachgebrauchs im häuslichen Umfeld.

Tab. 2 Zusammensetzung der Schülerinnen- und Schülerstichprobe $(N=409)$

\begin{tabular}{lll}
\hline Subgruppen der Stichprobe & Absoluter Anteil & Relativer Anteil (\%) \\
\hline Lebensweltlich einsprachig & 191 & 46,7 \\
Lebensweltlich mehrsprachig & 218 & 53,3 \\
Gesamt & 409 & 100 \\
\hline
\end{tabular}




\section{Ergebnisse}

\subsection{Wirksamkeit der Intervention}

Der Vergleich zwischen der Sprachbewusstheit von Experimental- und Kontrollgruppe zu MZP 2 mittels Kovarianzanalyse zeigt, dass Schülerinnen und Schüler der Experimentalgruppe höhere Sprachbewusstheitswerte $(M=12,38, S D=4,53)$ als die der Kontrollgruppe $(M=10,65, S D=4,32)$ erreichen. Kontrolliert man für beide Gruppen Sprachkompetenz und allgemeine kognitive Fähigkeiten, so ist dieser Unterschied signifikant $\left(F(1)=22,06, p<0,001, \eta^{2}\right.$ part $\left.=0,052\right)$. Der Vorsprung der Experimentalgruppe zeigt sich ebenso in den Subgruppen lebensweltlich mehrsprachig vs. lebensweltlich einsprachig. Mehrsprachige Schülerinnen und Schüler der Experimentalgruppe schneiden besser ab als mehrsprachige Schülerinnen und Schüler der Kontrollgruppe ( $\mathrm{M}=12,24, \mathrm{SD}=4,17$ für Mehrsprachige der Experimentalgruppe; $\mathrm{M}=11,34, \mathrm{SD}=4,74$ für Mehrsprachige der Kontrollgruppe). Unter der Kontrolle von Sprachkompetenz und allgemeinen kognitiven Fähigkeiten nähert sich der Unterschied der Signifikanzgrenze $(\mathrm{F}(1,407)=3,627, p=0,058)$. Ein ähnliches Ergebnis zeigt sich bei lebensweltlich einsprachigen Schülerinnen und Schülern der Experimental- und Kontrollgruppe. Erstere zeigen signifikant höhere Sprachbewusstheitswerte verglichen mit der Kontrollgruppe. Der Vorsprung der Experimentalgruppe bleibt auch sechs Monate nach der Intervention stabil $(F(1,407)=416,70, p<0,001$, $\left.\eta_{\text {part }}^{2}=0,506\right)($ Abb. 2).

Damit kann die erste Hypothese, dass die Experimentalgruppe höhere Sprachbewusstheitswerte erreicht als die Kontrollgruppe, als bestätigt angesehen werden. Der zu MZP 2 messbare Vorsprung der lebensweltlich mehrsprachigen Schülerinnen und Schüler der Experimentalgruppe im Vergleich zu den lebensweltlich mehrspra-

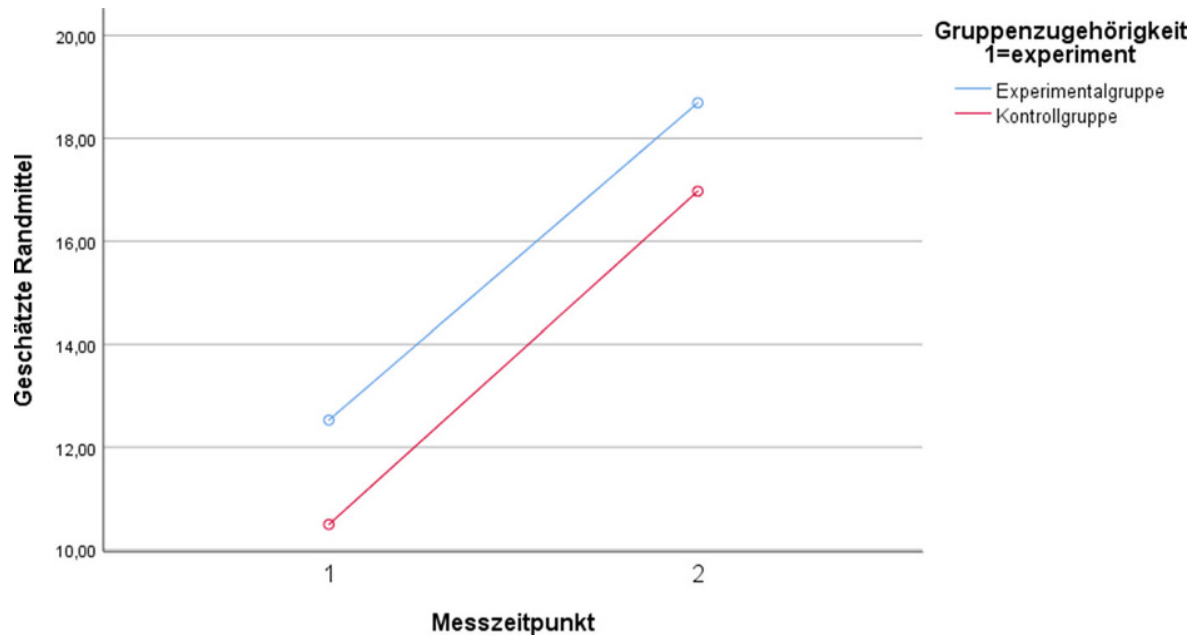

Abb. 2 Sprachbewusstheit in der Experimental- und Kontrollgruppe. (Die Kovariaten im Modell werden anhand der folgenden Werte berechnet: Faktorregressionswerte für schriftliche Sprachkompetenz1 $=0,0439513$ ) 
chigen der Kontrollgruppe ist zwar auch im Follow-up messbar, allerdings erreicht er nicht mehr die Signifikanzgrenze $(F(1,215)=2,16, p=0,143)$. In der Subgruppe der lebensweltlich einsprachigen Schülerinnen und Schüler zeigen sich hingegen, wie es bereits zu MZP 2 der Fall war, signifikante Unterschiede in der Sprachbewusstheit unter Kontrolle von Sprachkompetenz und allgemeinen kognitiven Fähigkeiten zwischen der Experimental- und Kontrollgruppe zum Vorteil der ersteren $\left(F(1,188)=5,36, p=0,022, \eta_{\text {part }}^{2}=0,028\right)$.

\subsection{Mehrsprachigkeit und Sprachbewusstheit}

Der Vergleich zwischen lebensweltlich einsprachigen und mehrsprachigen Schülerinnen und Schülern innerhalb der Experimentalgruppe zeigt sowohl im Posttest als auch im Follow-up keine Unterschiede für die Variable Sprachbewusstheit $(F(1,407)=0,098, p=0,754)$. Beide Subgruppen erreichen nahezu identische Sprachbewusstheitswerte (vgl. Abb. 3).

In der Kontrollgruppe zeichnet sich ein anderes Bild ab: Lebensweltlich mehrsprachige Schülerinnen und Schüler zeigen im Verlauf der Zeit eine konstant signifikant höhere Sprachbewusstheit als lebensweltlich einsprachige $(\mathrm{F}(1,194)=5,08$, $p=0,025, \eta^{2}$ part $\left.=0,026\right)$, wenn man ihre Sprachkompetenz und ihre allgemeinen kognitiven Fähigkeiten kontrolliert (Abb. 4). Auch hier ist sowohl bei den allgemeinen kognitiven Fähigkeiten $\left(\mathrm{F}(1,194)=9,90, p=0,002, \eta^{2}\right.$ part $\left.=0,049\right)$ als auch der Sprachkompetenz $\left(\mathrm{F}(1,194)=5,83, p=0,002, \eta^{2}\right.$ part $\left.=0,029\right)$ ein signifikanter Einfluss auf Sprachbewusstheit festzustellen.

Die zweite Hypothese, dass von einem sprachenintegrativen Unterricht lebensweltlich mehrsprachige Schülerinnen und Schüler in besonderem Maße profitieren, findet somit keine Bestätigung. In der Experimentalgruppe zeigt sich kein Vorsprung

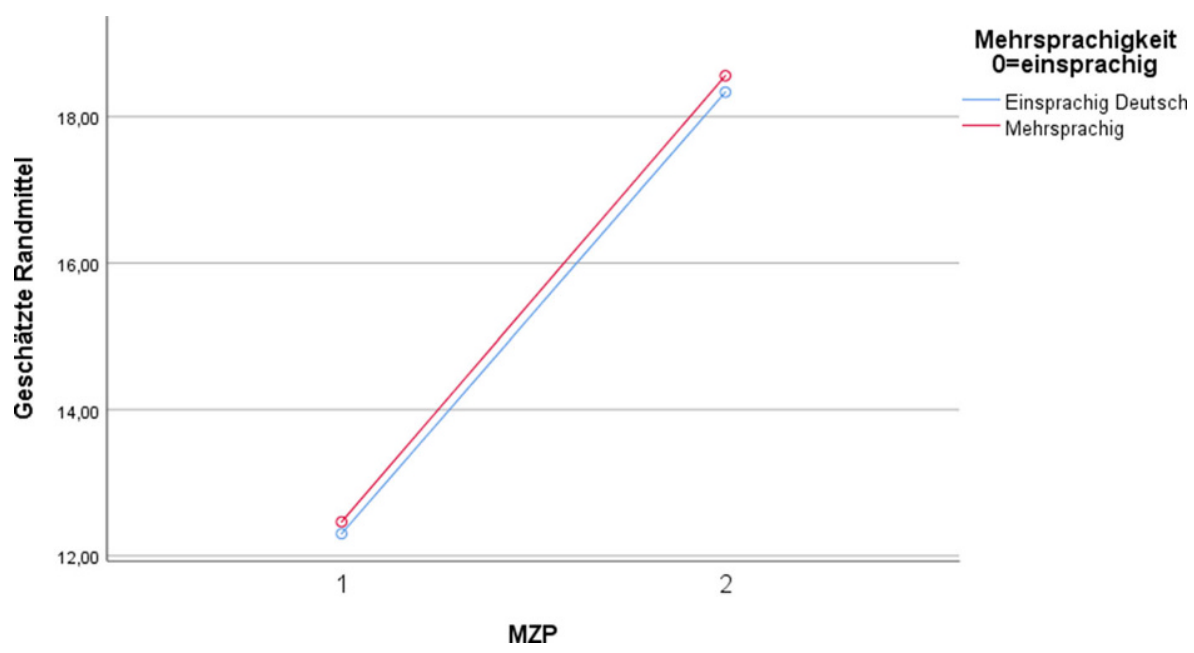

Abb. 3 Sprachbewusstheit ein- und mehrsprachiger Schülerinnen und Schüler in der Experimentalgruppe. (Die Kovariaten im Modell werden anhand der folgenden Werte berechnet: Faktorregressionswerte für schriftliche Sprachkompetenz1 = -0,0997778, Allgemeine kognitive Fähigkeit des Kindes = 100,73) 


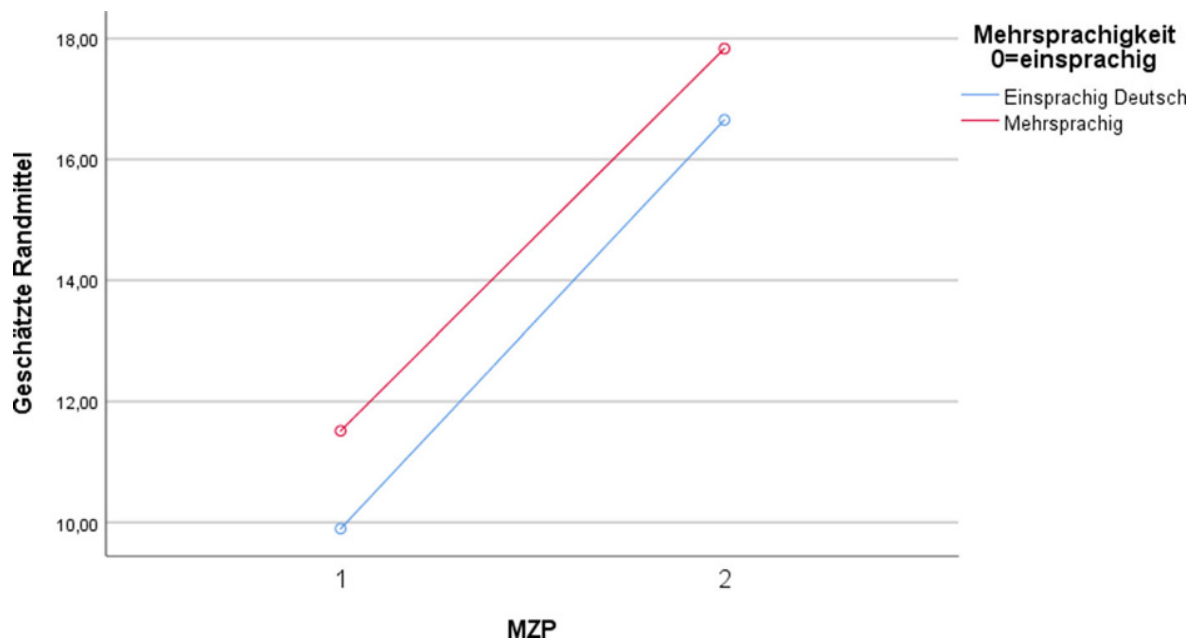

Abb. 4 Sprachbewusstheit ein- und mehrsprachiger Schülerinnen und Schüler der Kontrollgruppe

von lebensweltlich mehrsprachigen Schülerinnen und Schülern. Vielmehr deutet die Datenlage darauf hin, dass die lebensweltlich Einsprachigen im stärkeren Maße von der Intervention profitieren.

\subsection{Entwicklung von Sprachbewusstheit in der zweiten Hälfte der Grundschule}

Im Vergleich zwischen den MZP 2 und 3 zeigt sich in den beiden Gruppen ein starker und signifikanter Anstieg des globalen Sprachbewusstheitswerts $(t(197)=-15,083$, $p<0,001$ für die Kontrollgruppe und $(t(211)=-13.835, p<0,001$ für die Experimentalgruppe [Tab. 3]).

Das Ergebnis deutet darauf hin, dass sich Sprachbewusstheit in der zweiten Hälfte der Grundschulzeit sowohl im Zuge des regulären Deutschunterrichts als auch durch einen sprachenintegrativen Deutschunterricht entwickelt. Dass hierbei die sprachliche und kognitive Entwicklung der Schülerinnen und Schüler eine Rolle spielt, wurde bereits im Projekt „Sprachkompetenzen und Sprachbewusstheit“ gezeigt (Akbulut et al. 2017). Auch in der vorliegenden Studie zeigen sich signifikante, aber schwache Korrelationen zwischen Sprachkompetenzen im Deutschen, kognitiven Grundfähigkeiten und Sprachbewusstheit (Tab. 4).

Dieser Befund deutet darauf hin, dass sowohl die Sprachkompetenzen in der Umgebungssprache Deutsch als auch die allgemeinen kognitiven Grundfähigkei-

Tab. 3 Entwicklung von Sprachbewusstheit zum Ende der Grundschule

\begin{tabular}{|c|c|c|c|c|c|c|}
\hline \multicolumn{7}{|c|}{ Entwicklung von Sprachbewusstheit } \\
\hline \multirow[b]{2}{*}{ MZP } & \multicolumn{3}{|c|}{ Experimentalgruppe } & \multicolumn{3}{|c|}{ Kontrollgruppe } \\
\hline & M & SD & $N$ & M & SD & $N$ \\
\hline MZP 2 & 12,38 & 4,53 & 211 & 10,64 & 4,32 & 198 \\
\hline MZP 3 & 18,47 & 6,51 & 211 & 17,20 & 6,38 & 198 \\
\hline
\end{tabular}


Tab. 4 Korrelationen zwischen Sprachbewusstheit, Sprachkompetenzen und kognitiven Grundfähigkeiten

\begin{tabular}{lll}
\hline & Sprachkompetenzen & Kognitive Grundfähigkeiten \\
\hline Sprachbewusstheit MZP 2 & $r=178, p=0,000$ & $r=301, p=0,000$ \\
Sprachbewusstheit MZP 3 & $r=206, p=0,000$ & $r=274, p=0,000$ \\
\hline
\end{tabular}

ten zwar einen Einfluss auf die Entwicklung von Sprachbewusstheit am Ende der Grundschule ausüben, dieser jedoch zu gering ist, um die Entwicklung dieser Fähigkeit ausreichend erklären zu können. Schülerinnen und Schüler in verschiedenen Grundschulen zeigen sowohl in der Experimental- als auch in der Kontrollgruppe im Hinblick auf die Entwicklung von Sprachbewusstheit unterschiedliche Verläufe. Während in einigen Grundschulen bereits zu MZP 2 hohe Durchschnittswerte für Sprachbewusstheit erreicht werden, sind die Werte in anderen Grundschulen um mehr als fünf Punkte niedriger. Auch die Entwicklung von Sprachbewusstheit vom 2. zum 3. MZP zeigt unterschiedliche Verläufe. Folglich ist anzunehmen, dass neben der Zugehörigkeit zur Experimental- und Kontrollgruppe sowie die bereits erwähnten Faktoren (Sprachkompetenzen, kognitive Fähigkeiten) weitere Faktoren hinzukommen, die die Entwicklung von Sprachbewusstheit am Ende der Grundschulzeit beeinflussen.

\section{Diskussion}

In der vorliegenden Studie zeigen sich Effekte eines sprachenintegrativen Deutschunterrichts auch noch sechs Monate nach der Intervention. Schülerinnen und Schüler der Experimentalgruppe zeigen signifikant höhere Sprachbewusstheitswerte als Schülerinnen und Schüler der Kontrollgruppe. Innerhalb der Experimentalgruppe sind in Bezug auf Sprachbewusstheit keinerlei Unterschiede zwischen lebensweltlich ein- und mehrsprachigen Schülerinnen und Schülern festzustellen, während die entsprechenden Unterschiede in der Kontrollgruppe zugunsten der lebensweltlich Mehrsprachigen statistisch signifikant sind. Es lässt sich vermuten, dass Mehrsprachige durch das Aufwachsen mit mehreren Sprachsystemen über eigene Ressourcen zum Sprachvergleich verfügen, sodass das externe Bereitstellen solcher Ressourcen im Deutschunterricht kein signifikantes Wachstum von Sprachbewusstheit nach einer relativ kurzen Intervention fördert. Für lebensweltlich einsprachige Schülerinnen und Schüler, die für den Sprachvergleich unterrichtlicher Unterstützung bedürfen, scheint hingegen bereits eine relativ kurze Intervention ein signifikantes Wachstum von Sprachbewusstheit zu bewirken. Mehrsprachigkeit kann somit als eine interne Ressource für Mehrsprachige sowie als eine externe Ressource für Sprachbewusstheit (durch ihren Einbezug im Unterricht) angesehen werden. Gleichzeitig werfen die Ergebnisse neue Fragen auf, vor allem im Hinblick auf die Beteiligung anderer als der untersuchten Faktoren an der Entwicklung von Sprachbewusstheit zum Ende der Grundschulzeit. Durch die recht hohe Streuung der Sprachbewusstheitswerte zu den beiden Messzeitpunkten kann vermutet werden, dass die Unterrichtsgestaltung sowohl in der Experimental- als auch in der Kontrollgruppe als eine Einflussgrö- 
Be auf die Entwicklung von Sprachbewusstheit in Frage kommt. Hier sind weitere Studien notwendig.

\section{Limitationen}

Die Ergebnisse zu Sprachbewusstheit, wie sie in der vorliegenden Studie modelliert und operationalisiert wurde, sind stets vor dem Hintergrund der gewählten Methode zu interpretieren. Die Tatsache, dass die Erhebung von Sprachbewusstheit in Tandems geschah, beeinflusst möglicherweise die Ergebnisse auf der Schülerebene. Zwar wurde jedes Tandemmitglied gesondert zu einer metasprachlichen Äußerung aufgefordert und die Reihenfolge des Fragens variiert, nichtsdestotrotz kann eine geringe gegenseitige Beeinflussung nicht ausgeschlossen werden.

\section{Fazit}

Mit der Interventionsstudie konnte belegt werden, dass ein sprachenintegrativer Deutschunterricht, in dessen Rahmen Mehrsprachigkeit zur Sprachreflexion und zum Sprachvergleich genutzt wird, zu einer Erhöhung der Sprachbewusstheit führt. Dieses Resultat ist von hoher Relevanz, wenn es um Fragen schulischer Sprachbildung geht. Es zeigt, dass sowohl lebensweltlich mehrsprachige als auch einsprachige Lernerinnen und Lerner bereits in der Grundschule von einem sprachenintegrativen Deutschunterricht profitieren können. Gleichzeitig zeigen die Daten, dass sich Sprachbewusstheit auch im regulären Deutschunterricht von Klasse drei zu Klasse vier signifikant entwickelt. Dies verwundert nicht, wenn man die in der Forschung mehrfach belegten, und auch in der vorliegenden Untersuchung festgestellten, Zusammenhänge zwischen Sprachkompetenzen, kognitiven Grundfähigkeiten und Sprachbewusstheit berücksichtigt. Mit Blick auf die in der Forschung kontrovers diskutierte Frage nach einem Zusammenhang zwischen Mehrsprachigkeit und Sprachbewusstheit kommt die vorliegende Studie zu einem unerwarteten Ergebnis, das gleichzeitig einen Anknüpfungspunkt für weitere Untersuchungen darstellt: Während in der Kontrollgruppe lebensweltlich mehrsprachige Schülerinnen und Schüler unter Kontrolle der kognitiven Fähigkeiten und der Sprachkompetenz im Deutschen einen Vorsprung in Sprachbewusstheit haben, lassen sich in der Experimentalgruppe weder direkt nach der Intervention noch im Follow Up Vorteile messen. Dies kann als ein Indiz dafür gewertet werden, dass sprachenintegrativer Deutschunterricht insbesondere lebensweltlich einsprachig aufwachsenden Schülerinnen und Schülern die Möglichkeit bietet, ihre Sprachbewusstheit auszubauen.

Funding Open Access funding enabled and organized by Projekt DEAL.

Open Access Dieser Artikel wird unter der Creative Commons Namensnennung 4.0 International Lizenz veröffentlicht, welche die Nutzung, Vervielfältigung, Bearbeitung, Verbreitung und Wiedergabe in jeglichem Medium und Format erlaubt, sofern Sie den/die ursprünglichen Autor(en) und die Quelle ordnungsgemäß nennen, einen Link zur Creative Commons Lizenz beifügen und angeben, ob Änderungen vorgenommen wurden. 
Die in diesem Artikel enthaltenen Bilder und sonstiges Drittmaterial unterliegen ebenfalls der genannten Creative Commons Lizenz, sofern sich aus der Abbildungslegende nichts anderes ergibt. Sofern das betreffende Material nicht unter der genannten Creative Commons Lizenz steht und die betreffende Handlung nicht nach gesetzlichen Vorschriften erlaubt ist, ist für die oben aufgeführten Weiterverwendungen des Materials die Einwilligung des jeweiligen Rechteinhabers einzuholen.

Weitere Details zur Lizenz entnehmen Sie bitte der Lizenzinformation auf http://creativecommons.org/ licenses/by/4.0/deed.de.

\section{Literatur}

Akbulut, M., Bien-Miller, L., \& Wildemann, A. (2017). Mehrsprachigkeit als Ressource für Sprachbewusstheit. Zeitschrift für Grundschulforschung, 2017(02), 61-74.

Andresen, H. (1985). Schriftspracherwerb und die Entstehung von Sprachbewusstheit. Opladen: Westdeutscher Verlag.

Andronie, M., Krzyzek, S., Bien-Miller, L., \& Wildemann, A. (2019). Theory and practice: from Delphistudy to pedagogical training. Qualitative Research Journal. https://doi.org/10.1108/QRJ-03-20190031.

Bialystok, E. (1986). Factors in the growth of linguistic awareness. Child Development, 57(2), 498-510.

Bialystok, E. (1987). Words as things: development of word concept by bilingual children. Studies in Second Language Acquisition, 9(2), 133-140.

Bialystok, E. (1988). Levels of bilingualism and levels of linguistic awareness. Developmental Psychology, 24, 560-567.

Bialystok, E. (1991). Metalinguistic dimensions of bilingual language proficiency. In E. Bialystok (Hrsg.), Language processing in bilingual children (S. 113-140). London: Cambridge University Press.

Bialystok, E. (1999). Cognitive complexity and attentional control in the bilingual mind. Child Development, 70(3), 636-644.

Bialystok, E. (2001). Bilingualism in development: language, literacy \& cognition. New York: Cambridge University Press.

Bialystok, E. (2017). The bilingual adaptation: how minds accommodate experience. Psychological Bulletin, 143(3), 233-262.

Bialystok, E., \& Ryan, E. B. (1985). Toward a definition of metalinguistic skill. Merill-Palmer Quarterly, 31(3), 229-251.

Bien-Miller, L., \& Wildemann, A. (2020). Mehrsprachigkeit als Ressource für Sprachbetrachtung nutzen: (K)ein Konsens zwischen Theorie und Praxis möglich? Der Deutschunterricht, 2020(2), 62-70.

Budde, M. (2012). Über Sprache reflektieren: Unterricht in sprachheterogenen Lerngruppen. Kassel: Kassel University Press.

Busch, B. (2016). Mehrsprachigkeit. Wien: Facultas wuv/utb.

Cromdal, J. (1999). Childhood bilingualism and metalinguistic skills: analysis and control in young Swedish-English bilinguals. Applied Psycholinguistics, 20, 1-20.

Eichler, W. (2007). Sprachbewusstheit. In B. Beck \& E. Klieme (Hrsg.), Sprachliche Kompetenzen: Konzepte und Messung: DESI-Studie (Deutsch Englisch Schülerleistung International) (S. 147-157). Weinheim: Beltz.

Ellis, R. (2008). The study of second language acquisition. Oxford: Oxford University Press.

Esser, H. (2006). Sprache und Integration. Sie sozialen Bedingungen und Folgen des Spracherwerbs von Migranten. Frankfurt, New York: Campus.

Fehling, S. (2005). Language Awareness und bilingualer Unterricht: Eine komparative Studie. Frankfurt am Main: Peter Lang Editions.

Galambos, S. J., \& Goldin-Meadow, S. (1990). The effects of learning two languages on levels of metalinguistic awareness. Cognition, 34, 1-56.

Galambos, S. J., \& Hakuta, K. (1988). Subject-specific and task-specific characteristics of metalinguistic awareness in bilingual children. Applied Psycholinguistics, 9, 141-162.

García, O. (2009). Bilingual education in the 21st century: a global perspective. Malden/Oxford: Basil/ Blackwell.

Gombert, J.E. (1992). Metalinguistic development. London: Harvester Weatsheaf.

Gornik, H. (1989). Metasprachliche Entwicklung bei Kindern: Definitionsprobleme und Forschungsergebnisse - ein Überblick. Osnabrücker Beiträge zur Sprachtheorie, 40, 39-57. 
Gornik, H. (2014). Sprachreflexion, Sprachbewusstheit, Sprachwissen, Sprachgefühl und die Kompetenz der Sprachthematisierung - ein Einblick in ein Begriffsfeld. In H. Gornik (Hrsg.), Sprachreflexion und Grammatikunterricht (S. 41-58). Baltmannsweiler: Schneider Verlag Hohengehren.

Gornik, H. (2010). Über Sprache reflektieren: Sprachthematisierung und Sprachbewusstheit. In V. Frederking \& H.-W. Huneke, et al. (Hrsg.), Sprache und Medien. Taschenbuch des Deutschunterrichts, Bd. 2. Baltmannsweiler: Schneider Verlag Hohengehren.

Grosjean, F. (2020). Individuelle Zwei- und Mehrsprachigkeit. In I. Gogolin, A. Hansen, S. McMonagle \& D. Rauch (Hrsg.), Handbuch Mehrsprachigkeit und Bildung (S. 13-21). Wiesbaden: Springer VS.

Hakes, D. T. (1980). The development of metalinguistic abilities in children. Berlin: Springer.

Hattie, J. A., Biggs, J., \& Purdie, N. (1996). Effects of learning skills interventions on student learning: a meta-analysis. Review of Educational Research, 66, 99-136.

Hawkins, E. (1984). Awareness of language. Cambridge: Cambridge University Press.

Hopp, H., Kieseier, T., Vogelbacher, M., Köser, S., \& Thoma, D. (2017). Mehrsprachigkeit und Metasprachliche Bewusstheit im Englischerwerb in der Grundschule. In I. Fuchs, S. Jeuk \& W. Knapp (Hrsg.), Mehrsprachigkeit: Spracherwerb, Unterrichtsprozesse, Schulentwicklung: Beiträge zum 11. Workshop „,Kinder und Jugendliche mit Migrationshintergrund“, 2015 (S. 55-74). Stuttgart: Klett.

Hornberger, N.H., \& Holly, H. (2012). Translanguaging and transnational literacies in multilingual classrooms: a biliteracy lens. International Journal of Bilingual Education and Bilingualism, 15(3), 261-278.

James, C., \& Garrett, P. (1992). Language awareness in the classroom. London/New York: Longman.

Jessner, U. (2007). Language awareness in multilinguals. In J. Cenoz \& N. Hornberger (Hrsg.), Knowledge about language (S. 357-369). New York: Springer.

Karmiloff-Smith, A. (1986). From meta-processes to conscious access: evidence from children's metalinguistic and repair data. Cognition, 23(2), 95-147.

Karmiloff-Smith, A. (1992). Beyond modularity: a developmental perspective on cognitive science. Massachusetts: MIT Press.

Klieme, E., \& Rakoczy, K. (2008). Empirische Unterrichtsforschung und Fachdidaktik: Outcome-orientierte Messung und Prozessqualität des Unterrichts. Zeitschrift für Pädagogik, 54(2), 222-237.

Krafft, A. (2014). Zur Entwicklung metasprachlicher Fähigkeiten bei Kindern mit ein- und mehrsprachigem Hintergrund. Baltmannsweiler: Schneider Verlag Hohengehren.

Kurtz, G., Hofmann, N., Biermas, B., Back, T., \& Haseldiek, K. (2014). Sprachintensiver Unterricht: Ein Handbuch. Baltmannsweiler: Schneider Verlag Hohengehren.

Kuyumcu, R. (2014). Sprach(en)entwicklung und Sprachreflexion: Drei Fallstudien zu zweisprachig aufwachsenden Vorschulkindern mit Erstsprache Türkisch und Zweitsprache Deutsch. Tübingen: Stauffenburg.

Marinova-Todd, S. H., Siegel, L., \& Mazabel, S. (2013). The association between morphological awareness and literacy in English language learners from different language backgrounds. Topics in Language Disorders, 33(1), 93-107.

Mimeau, C., Laroche, L., \& Deacon, S. H. (2019). The relation between syntactic awareness and contextual facilitation in word reading: What is the role of semantics? Journal of Research in Reading, 42(1), $178-192$.

Mokhtari, K., \& Thompson, B.H. (2006). How problems of reading fluency and comprehension are related to difficulties in syntactic awareness skills among fifth graders. Reading Research and Instruction, 46(1), 73-94.

Oomen-Welke, I. (2010). Didaktik der Sprachenvielfalt. In B. Ahrenholz \& I. Oomen-Welke (Hrsg.), Deutsch als Zweitsprache (2. Aufl. S. 479-492). Baltmannsweiler: Schneider Verlag Hohengehren.

Oomen-Welke, I. (2017). Didaktik der Sprachenvielfalt. In B. Ahrenholz \& I. Oomen-Welke (Hrsg.), Deutsch als Zweitsprache (4. Aufl. S. 617-632). Baltmannsweiler: Schneider Verlag Hohengehren.

Reich, H.-H., \& Krumm, H.-J. (2013). Sprachbildung und Mehrsprachigkeit: Ein Curriculum zur Wahrnehmung und Bewältigung sprachlicher Vielfalt im Unterricht. Münster: Waxmann.

Reich, H.H., Roth, H.-J., \& Gantefort, C. (2008). Der Sturz ins Tulpenbeet: Deutsche Sprachversion: Auswertungsbogen und Auswertungshinweise. In T. Klinger, K. Schwippert \& B. Leiblein (Hrsg.), Evaluation im Modellprogramm FÖRMIG (S. 209-237). Münster: Waxmann.

Ricciardelli, L. A. (1992). Bilingualism and cognitive development in relation to threshold theory. Journal of Psycholinguistic Research, 21(4), 301-316.

Rösch, H. (2014). BeFo und die Folgen für die DaZ-Didaktik. In B. Lütke \& I. Petersen (Hrsg.), Deutsch als Zweitsprache: erwerben, lernen und lehren: Beiträge aus dem 9. Workshop „Kinder mit Migrationshintergrund" 2013 (S. 195-208). Stuttgart: Fillibach.

Rösch, H. (2017). Deutschunterricht in der Migrationsgesellschaft: Eine Einführung. Stuttgart: Metzler. 
Schöler, H. (1987). Zur Entwicklung metasprachlichen Wissens. In Deutsche Gesellschaft für Sprachheilpädagogik (Hrsg.), Spracherwerb und Spracherwerbsstörungen (S. 339-359). Hamburg: Wartenberg.

Svalberg, A. M.-L. (2012). Peer interaction, cognitive conflict and anxiety on a grammar awareness course for language teachers. Language Awareness, 21, 137-155.

Tragant, E., \& Victori, M. (2012). Language learning strategies, course grades and age in EFL secondary school learners. Language Awareness, 21(3), 293-308.

Ulrich, W. (2001). Grammatikunterricht - Wortschatzarbeit - Unterrichtsmittel. Didaktik der deutschen Sprache: Ein Arbeits- und Studienbuch in drei Bänden: Texte - Materialien - Reflexionen, Bd. 3. Stuttgart: Klett.

Waller, M. (1988). Komponenten der metasprachlichen Entwicklung und Bedingungen ihres ontogenetischen Aufbaus. Zeitschrift für Entwicklungspsychologie und Pädagogische Psychologie, 20(4), 297-321.

Weiß, R. (2005). CFT 20-R: Grundintelligenztest Skala 2 - Revision. Göttingen: Hogrefe.

Wildemann, A. (2013). Sprache(n) thematisieren - Sprachbewusstheit fördern. In S. Gailberger \& F. Wietzke (Hrsg.), Handbuch kompetenzorientierter Deutschunterricht: Diagnostizieren - Binnendifferenzieren - Fördern (S. 321-338). Weinheim/Basel: Beltz.

Wildemann, A., Akbulut, M., \& Bien-Miller, L. (2018). Mehrsprachige Sprachbewusstheit und deren Potenzial für den Grundschulunterricht. In G. Mehlhorn \& B. Brehmer (Hrsg.), Potenziale von Herkunftssprachen: Sprachliche und außersprachliche Einflussfaktoren (S. 117-143). Tübingen: Stauffenburg.

Wildemann, A., Bien, L., \& Akbulut, M. (2016). Mehrsprachige Sprachbewusstheit zum Ende der Grundschulzeit: Vorstellung und Diskussion eines Elizitationsverfahrens. Zeitschrift für Interkulturellen Fremdsprachenunterricht, 2016(2), 42-56.

Wildemann, A., Bien-Miller, L., \& Akbulut, M. (2020a). Mehrsprachigkeit und Sprachbewusstheit: empirische Befunde und Unterrichtskonzepte. In I. Gogolin, A. Hansen, S. McMonagle \& D. Rauch (Hrsg.), Handbuch Mehrsprachigkeit und Bildung (S. 119-123). Wiesbaden: Springer VS.

Wildemann, A., Andronie, M., Bien-Miller, L. \& Krzyzek, S. (2020b). Sprachliche Übergänge im Deutschunterricht (schaffen). Eine Interventionsstudie mit Grundschullehrerinnen und -lehrern. In M. Budde \& F. Prüsmann (Hrsg.), Vom Sprachkurs Deutsch als Zweitsprache zum Regelunterricht. Übergänge bewältigen, ermöglichen, gestalten. In der Reihe Deutsch als Zweitsprache. Positionen, Perspektiven, Potenziale. (S. 159-183). Münster: Waxmann.

Woolley, M.E., Bowen, G.L., \& Bowen, N. K. (2004). Cognitive pretesting and the developmental validity of child self-report instruments: theory and applications. Research on Social Work Practice, 14(3), 191-200. 